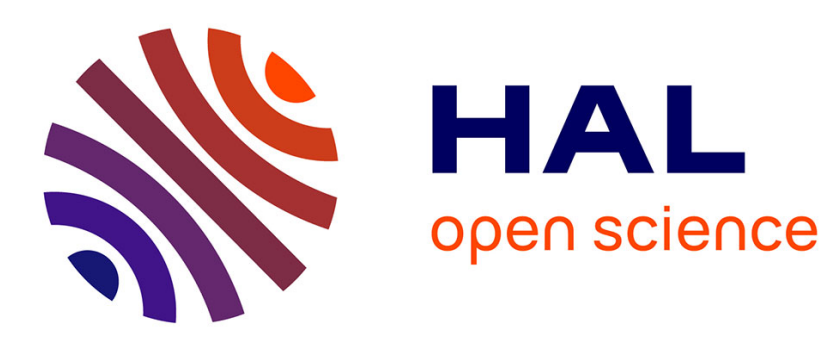

\title{
Paraxial ray-tracing approach for the simulation of ultrasonic inspection of welds
}

\author{
A. Gardahaut, K. Jezzine, D. Cassereau
}

\section{To cite this version:}

A. Gardahaut, K. Jezzine, D. Cassereau. Paraxial ray-tracing approach for the simulation of ultrasonic inspection of welds. AIP Conference Proceedings, 2014, 1581 (1), pp.529-536. 10.1063/1.4864865 . cea-01820681

\section{HAL Id: cea-01820681 https://hal-cea.archives-ouvertes.fr/cea-01820681}

Submitted on 15 Jan 2019

HAL is a multi-disciplinary open access archive for the deposit and dissemination of scientific research documents, whether they are published or not. The documents may come from teaching and research institutions in France or abroad, or from public or private research centers.
L'archive ouverte pluridisciplinaire HAL, est destinée au dépôt et à la diffusion de documents scientifiques de niveau recherche, publiés ou non, émanant des établissements d'enseignement et de recherche français ou étrangers, des laboratoires publics ou privés. 


\title{
Paraxial Ray-Tracing Approach for the Simulation of Ultrasonic Inspection of Welds
}

\author{
Audrey Gardahaut*, Karim Jezzine* and Didier Cassereau ${ }^{\dagger, * *}$ \\ ${ }^{*}$ CEA, LIST, Digiteo Labs, Bât 565, PC 120, F-91191, Gif-sur-Yvette, France \\ ${ }^{\dagger}$ CNRS, UMR 7623, LIP, 15 rue de l'école de médecine, 75006 Paris, France \\ ${ }^{* *}$ ESPCI ParisTech, 10 rue Vauquelin, 75005 Paris, France
}

\begin{abstract}
On-site inspection of bimetallic or austenitic welds can be very difficult to interpret owing to their internal structures. Skewing and splitting of the ultrasonic beam may occur due to the anisotropic and inhomogeneous properties of the welding material. In this paper, we present a ray-based method to simulate the propagation of ultrasonic waves in such structures. The formalism is based on dynamic ray tracing system in Cartesian coordinates along a reference ray. Standard ray tracing consists in the solution of a system of linear ordinary differential equations of the first order and is used to determine the trajectory of the ray. Likewise, dynamic ray tracing (DRT) also called paraxial ray tracing consists in the solution of an additional system of linear ordinary differential equations along the ray allowing paraxial quantities to be computed. It is used to evaluate the geometrical spreading and amplitude along the ray and in its vicinity. DRT is applied on a smooth representation of the elastic properties of the weld obtained thanks to an image processing technique applied on a macrograph of the weld. Simulation results are presented and compared to finite elements and experimental results.
\end{abstract}

Keywords: Dynamic Ray Tracing Method, Paraxial Rays, Weld Inspection, Anisotropic and Inhomogeneous media. PACS: 43.20.Dk - 46.40.Cd - 81.05.Xj- 07.05.Pj

\section{CONTEXT AND OBJECTIVES}

The interpretation of on-sites inspections of austenitic or bimetallic welds is particularly difficult due to their internal structures. Indeed, some disturbances of the beam, such as splitting and skewing [1], can be observed on experimental echoes. It has been highlighted that these disruptions are due to the anisotropic and inhomogeneous polycrystalline structure of the weld [2]. Simulation of ultrasonic inspection can help to understand these phenomena. Various models have been developed to simulate the ultrasonic propagation such as finite differences [3], finite element models [4, 5] or ray-tracing models [6]. A semi-analytical propagation model, based on Dynamic Ray Tracing model (DRT), has been implemented in the CIVA software [7, 8]. This model has been applied on a weld described as a set of several homogeneous domains with a constant crystallographic orientation. However, if the domains have small dimensions compared to the wavelength, the results are valid only if the contrast of impedance between two neighboring media is small. In some cases, the weld may be described as a continuously varying description of the crystallographic orientation. This paper describes the modeling approach and the developments made in order to apply the DRT model on a smooth cartography of the crystallographic orientation. To obtain this description, an image processing technique has been developed and applied on the original macrograph of a weld. The DRT model has been numerically validated in $2 \mathrm{D}$ by a comparison of the transmitted ultrasonic wave field evaluated with a finite elements model on a weld described thanks to an analytical law, and experimentally in 3D on a smooth cartography of the crystallographic orientation.

\section{IMAGE PROCESSING TECHNIQUE FROM THE MACROGRAPH}

The image processing technique developed in this study has been applied on the macrograph of a bimetallic weld composed of a ferritic steel and a stainless steel. The aim is to obtain a cartography of the crystallographic orientation. This cartography will subsequently be used as input data for an ultrasonic propagation model. The processing is composed of four steps performed thanks to the ImageJ and Matlab softwares. The first stage is to detect the external contours of the weld. This operation allows us to extract the entire region of the weld containing the buttering and the welded zone. Figure 1 represents the original macrograph of a bimetallic weld and the external detected contours. 


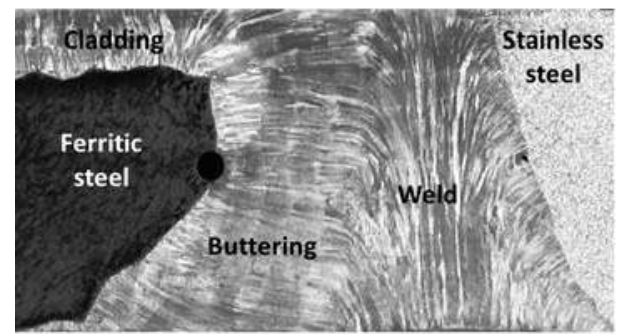

(a)

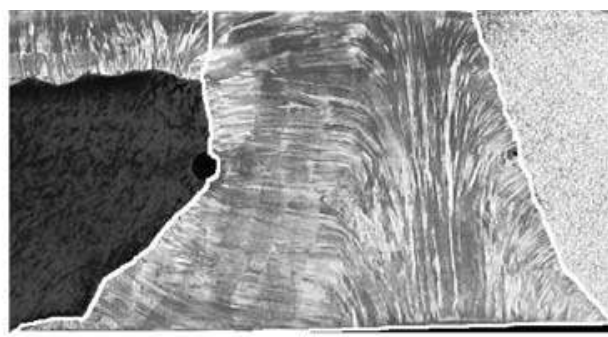

(b)

FIGURE 1. Representation of (a) the original macrograph of the studied weld and (b) its external detected contours.

The second step of the process is the calculation of the local orientation $\theta$ of the grains. This is done by using one of the functionalities of an ImageJ plug-in called OrientationJ [9]. Based on the evaluation of the structure tensor in local neighborhood, the plug-in determines the orientation of a region of interest. The algorithm consists in applying a Gaussian-shaped window on the image, then the structure tensor is evaluated for each pixel of the entire image by sliding this window. Finally, the program computes a colored image of the local orientation properties as shown in figure 2 .

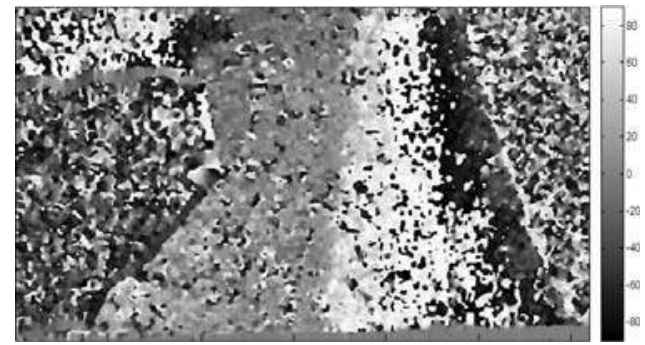

FIGURE 2. Representation in grayscale of the local orientation $\theta$ of the grains evaluated thanks to the OrientationJ plug-in [9].

As the DRT model is based on a high frequency approximation, the cartography of the local orientation has to be smooth. In Geophysics, Thierry et al. [10] have worked on obtaining a continuously varying description of the propagation velocity of the seismic waves in the ground. It has been realized by applying a smooth low-pass Gaussian filter on the representation of the velocity. The cut-off frequency of the filter is given by the relation:

$$
f(\mathbf{x})=\frac{1}{2 \pi \tau} \exp \left(-\frac{\mathbf{x}^{2}}{\tau^{2}}\right),
$$

where the correlation length $\tau$ is half of the wavelength. As we want to smooth the direction of the grains instead of the orientation, the filter is applied on the cosine and the sine of $2 \theta$. The images of the cosine and the sine of $2 \theta$ smoothed by a Gaussian filter with dimensions equal to the wavelength $\lambda$ (around $3 \mathrm{~mm}$ or 63 pixels) are shown in figure 3 .

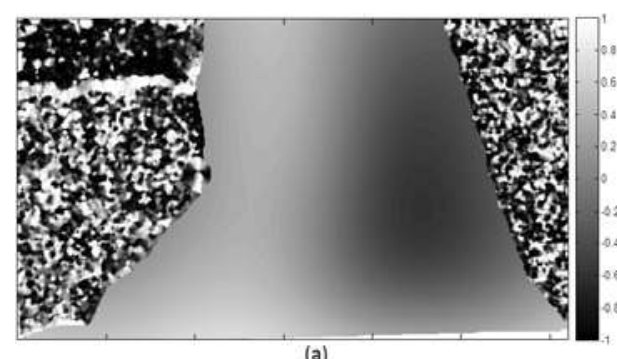

(a)

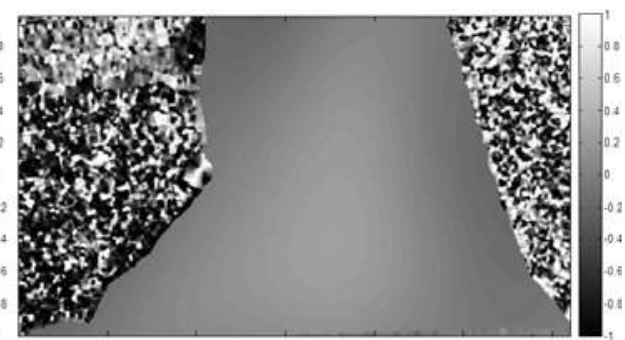

(b)

FIGURE 3. Smoothed representations of the (a) $\operatorname{cosine}(2 \theta)$ and (b) $\operatorname{sine}(2 \theta)$.

Finally, the cartography of the orientation $\theta$ is obtained from these two images (figure 4). 


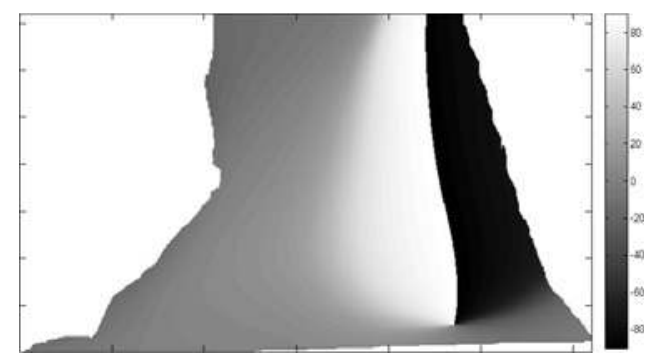

FIGURE 4. Representation of a smooth cartography of the crystallographic orientation of the studied weld.

\section{DYNAMIC RAY TRACING MODEL FOR A SMOOTH DESCRIPTION OF WELD}

The DRT model [11] has been applied to inhomogeneous media. This model is based on the evaluation of the ray trajectories and the travel-time and the computation of the amplitude of a ray tube during the propagation. To evaluate the ray-paths and travel-time, we have to solve the eikonal equation (2):

$$
(\nabla T)^{2}=\frac{1}{c(x)^{2}}
$$

representing a non-linear partial differential equation of the first order for the travel-time $T(x)$, where $c(x)$ is the phase velocity of the wave at position $x$. By deriving this equation, written in Hamiltonian form, a differential ray tracing system (3) called the axial ray system is expressed. This system is composed of two ordinary coupled differential equations describing the variation of the position $x_{i}$ and the slowness $p_{i}$ with respect to the travel-time:

$$
\left\{\begin{array}{l}
\frac{d x_{i}}{d T}=a_{i j k l} p_{l} g_{j}^{(m)} g_{k}^{(m)}=\mathscr{U}_{i}^{e(m)}, \\
\frac{d p_{i}}{d T}=-\frac{1}{2} \frac{\partial a_{j k l n}}{\partial x_{i}} p_{k} p_{n} g_{j}^{(m)} g_{l}^{(m)},
\end{array}\right.
$$

where $T$ is the travel-time, $a_{i j k l}$ the elasticity constants of the medium at the position $x_{i}$ normalized by the density $\rho$. The eigenvectors $g_{j}^{(m)}$ of the Christoffel tensor are the components of the polarization vector and $\mathscr{U}_{i}^{e(m)}$ is the energy velocity for the $m$ mode.

To describe the conservation of the energy inside the ray tube and compute the amplitude of a ray tube, the transport equation (4) has to be solved along a ray $\Omega$ in an anisotropic inhomogeneous medium:

$$
A(x) \nabla^{2} T(x)+2 \nabla A(x) \cdot \nabla T(x)=0 .
$$

It is a non-linear partial differential equation of the first order in scalar $A(x)$, the amplitude function. By deriving the axial ray system with respect to an initial parameter $\gamma$, we obtain a second system (5) of ordinary linear differential equations of the first order for the paraxial quantities $Q_{i}$ and $P_{i}$ :

$$
\left\{\begin{array}{c}
\frac{d}{d T}\left(\frac{\partial x_{i}}{\partial \gamma}\right)=\frac{d Q_{i}}{d T}=\frac{1}{2} \frac{\partial^{2} G_{m}}{\partial p_{i} \partial \gamma}=A_{i j} Q_{j}+B_{i j} P_{j}, \\
\frac{d}{d T}\left(\frac{\partial p_{i}}{\partial \gamma}\right)=\frac{d P_{i}}{d T}=-\frac{1}{2} \frac{\partial^{2} G_{m}}{\partial x_{i} \partial \gamma}=-C_{i j} Q_{j}-D_{i j} P_{j} .
\end{array}\right.
$$

$\gamma$ represents any parameter of the ray $\Omega$ and can be chosen, for example, as a take-off angle between a reference axis and the initial slowness vector. This system, called the paraxial ray system, is expressed with the parameter $G_{m}$ representing the normalized eigenvalues of the Christoffel tensor, written as $G_{m}=a_{i j k l} p_{j} p_{l} g_{i}^{(m)} g_{k}^{(m)}$. Three eigenvalues $G_{m}$ are evaluated, associated to three eigenvectors $g_{i}^{(m)}$ representing the three plane waves that propagate in the medium.

Axial and paraxial ray systems are solved simultaneously by using numerical techniques such as the Euler method in this case. The paraxial ray system (5) is written in a Cartesian regular coordinates system $x_{i}$ with $i, j=1,2,3$. It consists of six ordinary linear differential equations. It may be convenient to express this system in a wave front orthonormal coordinates system $y_{i}$ where the indexes $M$ and $N$ are equal to 1 or 2 [11]. The origin of this coordinate system moves along the ray $\Omega$ with the propagating wave front and the $y_{3}$-axis is orientated along the slowness vector 
$\vec{p}$ at the origin point. Axes $y_{1}$ and $y_{2}$ are mutually perpendicular in the plane tangent to the wave front at this origin point. The system consists then of four scalar linear differential equations.

The paraxial quantities $Q_{M}$ and $P_{M}$, with the index $M=1,2$, are the components of the vector $\vec{\Psi}$ describing a paraxial ray. $Q_{1}$ and $Q_{2}$ represent the spatial deviation of the paraxial ray from the axial one, and $P_{1}$ and $P_{2}$ are the slowness deviation of the paraxial ray from the axial ray as shown in figure 5 .

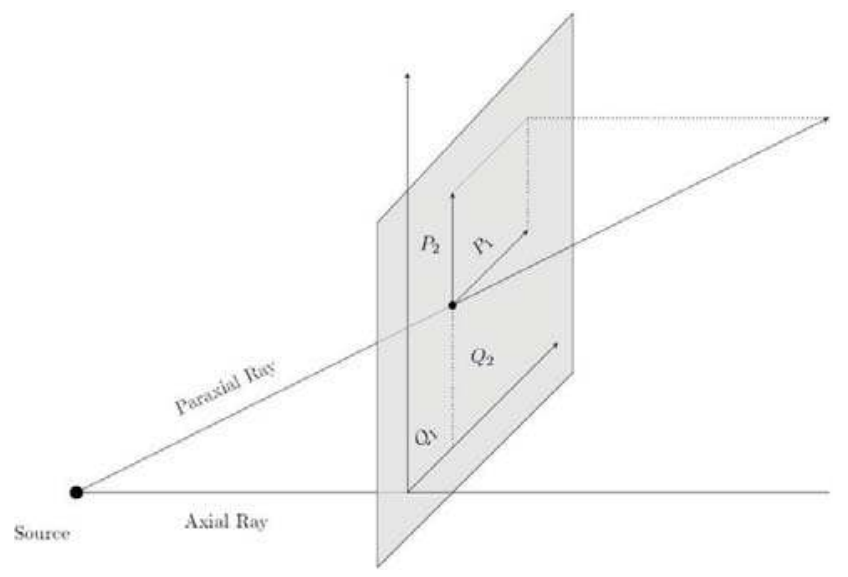

FIGURE 5. Representation of the paraxial quantities describing a paraxial ray.

The paraxial ray system is then written in a matrix form in the wave front orthonormal coordinates system (6) between two points:

$$
\left(\begin{array}{c}
Q_{M}^{(r+1)} \\
P_{M}^{(r+1)}
\end{array}\right)=\left(\begin{array}{ll}
\Pi_{11} & \Pi_{12} \\
\Pi_{21} & \Pi_{22}
\end{array}\right) \cdot\left(\begin{array}{c}
Q_{N}^{(r)} \\
P_{N}^{(r)}
\end{array}\right)=\left(\begin{array}{cc}
1+A_{M N} \Delta T & B_{M N} \Delta T \\
-C_{M N} \Delta T & 1-D_{M N} \Delta T
\end{array}\right) \cdot\left(\begin{array}{c}
Q_{N}^{(r)} \\
P_{N}^{(r)}
\end{array}\right)
$$

The paraxial quantities at iteration $(r+1)$ are written in function of the same quantities at $(r)$ through a $4 \times 4$ propagation matrix $\Pi$. Finally, the paraxial quantities at the last position are expressed in function of those at the first iteration through a propagation matrix $\Pi_{t o t}$ describing the complete propagation of the ultrasonic waves (7):

$$
\left(\begin{array}{c}
Q_{M}^{(n)} \\
P_{M}^{(n)}
\end{array}\right)=L_{(n)} \cdot L_{(n-1)} \ldots L_{(1)} \cdot L_{(0)} \cdot\left(\begin{array}{c}
Q_{N}^{(0)} \\
P_{N}^{(0)}
\end{array}\right)=\left(\begin{array}{cc}
\Pi_{11_{t o t}} & \Pi_{12_{t o t}} \\
\Pi_{21_{t o t}} & \Pi_{22_{t o t}}
\end{array}\right) \cdot\left(\begin{array}{c}
Q_{N}^{(0)} \\
P_{N}^{(0)}
\end{array}\right) .
$$

The evolution of the ray tube is represented in figure 6. At each time-step, the matrices $A_{M N}, B_{M N}, C_{M N}$ and $D_{M N}$ are re-evaluated so the propagation matrix is updated and the position and slowness vectors are computed from the axial ray system. Finally, the evolution of the ray tube between a source point and an observation point is expressed in

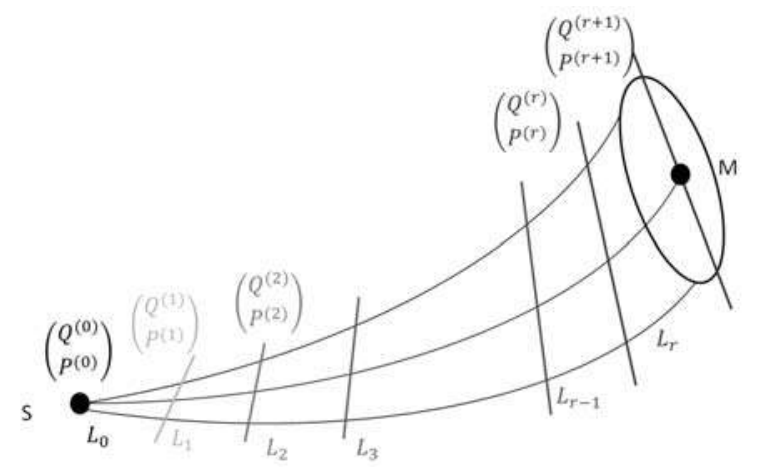

FIGURE 6. Representation of the evolution of a ray tube and its paraxial quantities during the propagation. 
3D through the geometrical spreading $\mathscr{L}(8)$ :

$$
\mathscr{L}=\left|\operatorname{det} \Pi_{12_{\text {tot }}}\right|^{1 / 2}
$$

\section{NUMERICAL VALIDATION ON AN ANALYTICAL DESCRIPTION}

The DRT model has been applied on a V-shaped weld on which the crystallographic orientation is described by a closed-form expression (9) proposed by Ogilvy [6]:

$$
\theta=\left\{\begin{array}{l}
\arctan \left(\frac{T(D+z * \tan \alpha)}{x^{n}}\right), \text { for } x \geq 0 \\
-\arctan \left(\frac{T(D+z * \tan \alpha)}{(-x)^{n}}\right), \text { for } x<0 .
\end{array}\right.
$$

Parameters $D$ and $\alpha$ describe the geometry of the weld when $T$ and $\eta$ express the evolution of the orientation of the grain. First, the ray trajectories have been compared to the literature in order to validate the resolution of the axial ray system. Figure 7 represents the ray trajectories evaluated by Connolly [12] in his thesis and with the DRT model in a V-weld whose parameters are: $D=2 \mathrm{~mm}, \alpha=21,80^{\circ}, T=1$ and $\eta=1$.

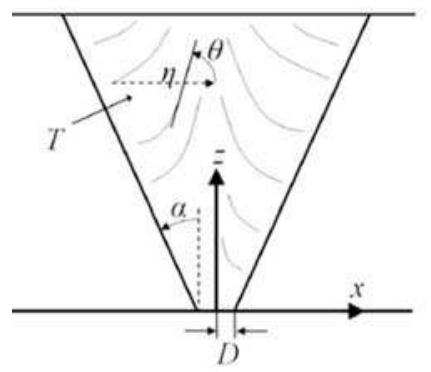

(a)

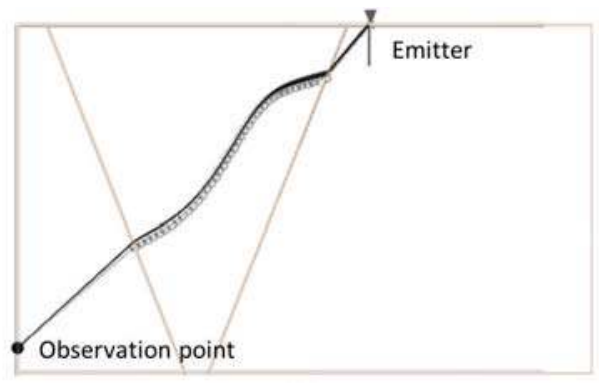

(b)

FIGURE 7. (a) Description of the parameters of the analytical law proposed by Ogilvy [6], (b) comparison of the ray trajectories evaluated by Connolly [12] (oo) and the DRT model (-).

The trajectories of a ray between a source point and an observation point evaluated with the dynamic ray tracing model are identical to those obtained by Connolly in his work. So, the axial ray system has been validated through the comparison of the ray trajectories with the literature. Then, the computation of the ray amplitudes has to be evaluated. To this aim, we have compared the ultrasonic wave field computed with the DRT model in this V-weld to results obtained with a hybrid code [13] involving a FE computation inside the weld. Results are shown in figure 8 . The comparison of the maximum particle velocity obtained with the DRT model applied on a smooth cartography of the crystallographic orientation shows an excellent agreement with those of the hybrid code.

\section{NUMERICAL AND EXPERIMENTAL VALIDATION ON A REALISTIC WELD}

\section{Numerical Validation}

Now that the DRT model has been validated in 2D on a simple description, we aim to apply it to a more realistic description of the crystallographic orientation (figure 9).

First, a comparison of the maximum particle velocity evaluated with the DRT model and the one obtained with the hybrid code is made (figure 10). The simulation has been done, on the cartography presented in figure 4, with an immersion probe with a $12,7 \mathrm{~mm}$ diameter emitting $\mathrm{L} 60^{\circ}$ waves at $2 \mathrm{MHz}$.

The comparison of the longitudinal wave field evaluated with both models (10) presents a very good agreement. Nevertheless, some differences are observed since the simulation with the DRT model has been made only for the 


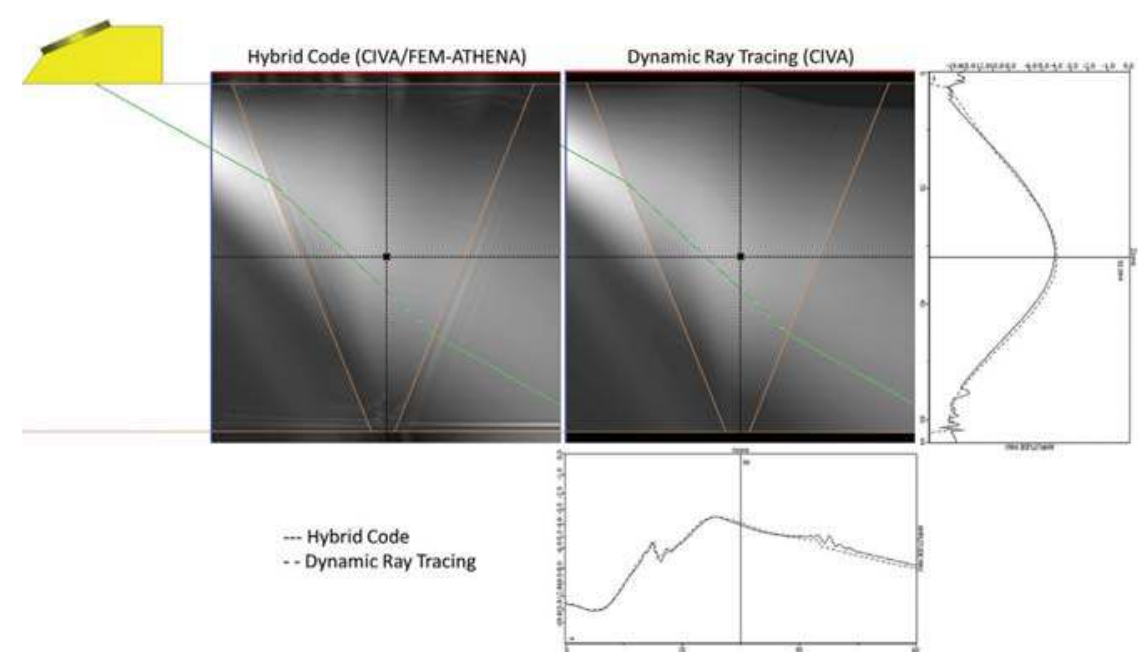

FIGURE 8. Representation of the maximum particle velocity for a weld described by a closed-form [6] and comparison of the results obtained with a 2D hybrid code and a DRT model in 2D.

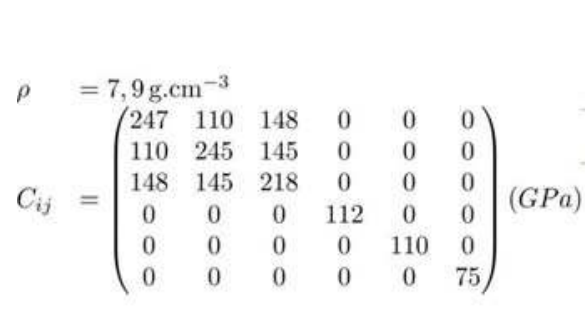

(a)

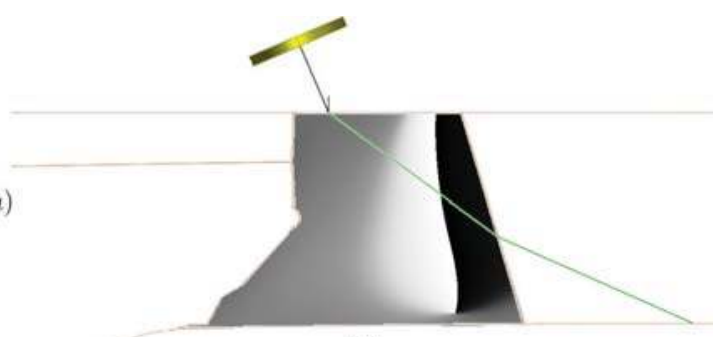

(b)

FIGURE 9. Application of the dynamic ray tracing model on a realistic weld: (a) physical properties of the weld, (b) configuration of the simulation.

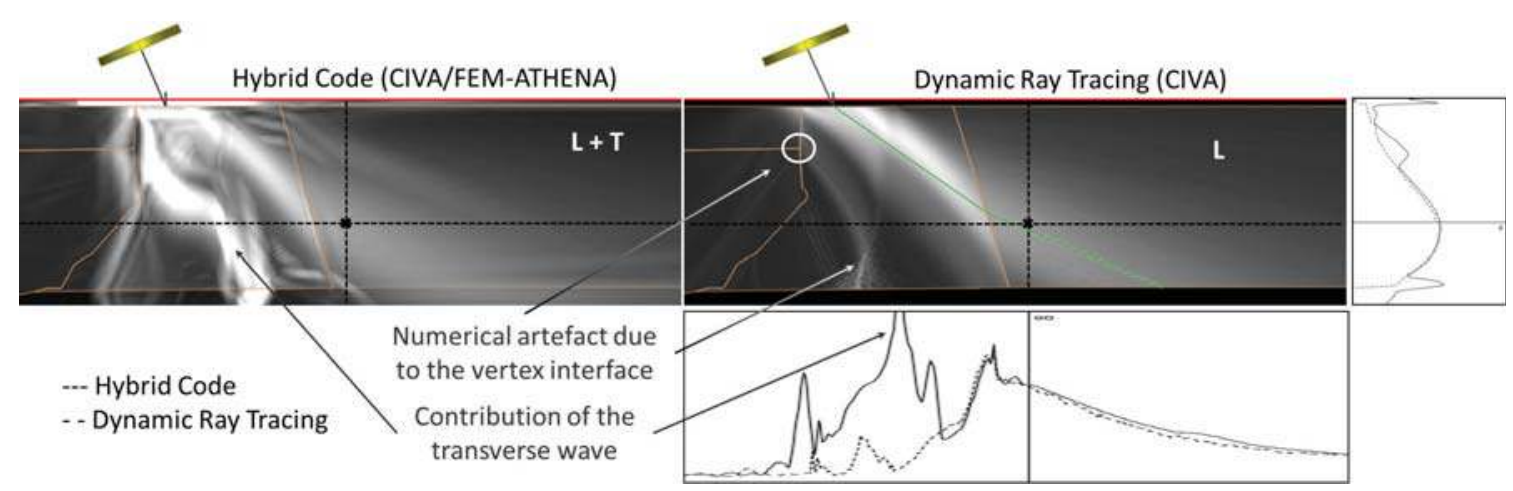

FIGURE 10. Representation of the maximum particle velocity for a weld described by a smooth cartography of the crystallographic orientation and comparison of the results obtained with a 2D hybrid code and a DRT model in 2D.

direct longitudinal wave while the hybrid code takes into account all the physical phenomenon such as the transverse wave, the reflections or the mode conversions. The beam of greater intensity on the wave field evaluated with the hybrid 
code corresponds to the transverse wave propagating in the weld. Furthermore, a beam is observed on the ultrasonic wave field evaluated with the DRT model. This beam is an artefact caused by the vertex interface between the weld and the cladding.

\section{Experimental Validation}

Experimental validation have also been performed on a mock-up of the weld used for the numerical validations. The acquisition has been realized with a $\mathrm{L} 60^{\circ}$ wedge probe with $12,7 \mathrm{~mm}$ diameter at $2 \mathrm{MHz}$ fixed on the weld. The 2D scanning in reception has been performed using a $0,2 \mathrm{~mm}$ needle hydrophone. The setup is shown in figure 11 .
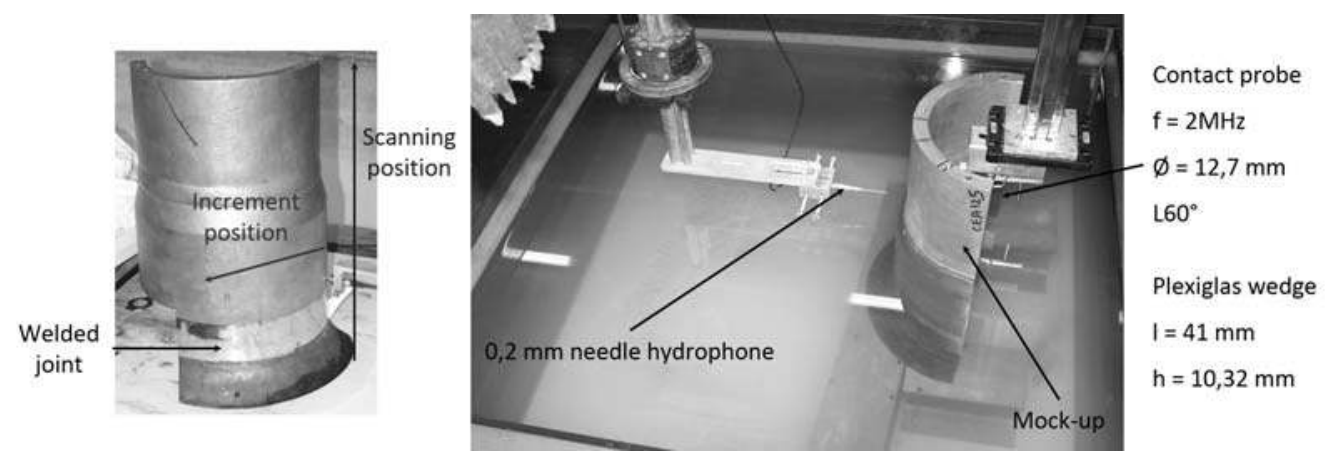

FIGURE 11. Illustration of the experimental setup.

This configuration has been reproduced in simulation in order to compare experimental and simulated evaluation of the ultrasonic longitudinal wave field. Results are shown in figure 12.
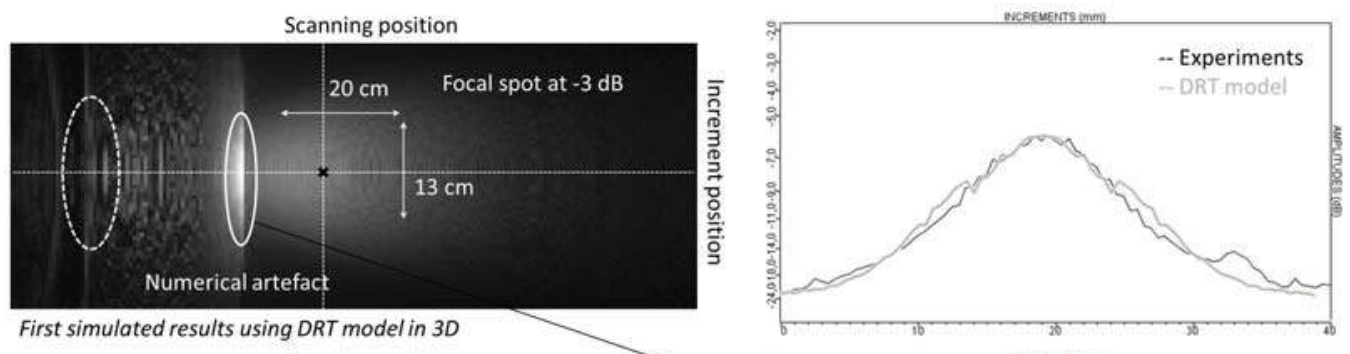

First simulated results using DRT model in 3D

Scanning position
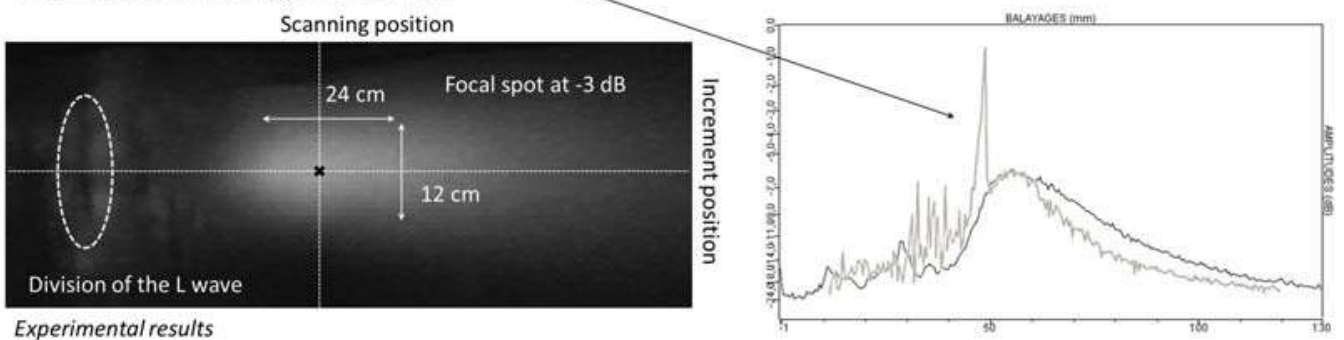

Experimental results

FIGURE 12. Comparison of the experimental and 3D computed transmitted wave field of the longitudinal wave.

The experimental and simulated results present a good agreement as shown in the representation of the wave field and on the superposition of the echodynamics. Furthermore, the dimensions of the longitudinal focal spot at $-3 \mathrm{~dB}$ are well evaluated with the DRT model. However, some numerical problems are shown in the simulated wave field. The high intensity contribution is a numerical artefact due to the interface. Indeed, it comes from the discontinuity of the normal at the interface between the weld and the stainless steel part. The region corresponding to the weld appear pixelated on the ultrasonic wave field. 
Because of an excessive degree of inhomogeneity in the cartography of the crystallographic orientation, the average geometrical spreading exponentially increases with the length of the rays. In the same time, the number of caustics also becomes large. This corresponds to a chaotic behavior of the rays [14], resulting in an inaccurate wave field computation. In order to overcome this problem, a work can be made to smooth the description thanks to the minimization of the Sobolev norm of slowness [15] or on the model by treating the problem of caustics with the Maslov method [16].

\section{CONCLUSION AND PERSPECTIVES}

This paper has presented the developments made to simulate the ultrasonic propagation in anisotropic and inhomogeneous media, such as welds, taking into account its physical internal properties. A dynamic ray tracing model, usually applied in geophysics, has been developed. Firstly, this model has been validated for a V-butt weld whose crystallographic orientation has been described by a closed-form expression. The ray trajectories have been successfully compared to the literature while the longitudinal wave field evaluated from these trajectories has been validated by comparison with results obtained with a hybrid code making a FE computation inside the weld. Then, both models have been applied on a smooth cartography of the crystallographic orientation. This description has been obtained by the application of an image processing technique on the macrograph of the weld. The steps of this process have been presented in this paper. Its aim was to obtain a smooth description compatible with the DRT model. The 2D simulations of the ultrasonic longitudinal wave field realized with both models have shown really good agreement. Then the DRT simulation in 3D has been successfully compared to experimental results. Nevertheless, some developments are expected to deal with numerical problems such as the chaotic behavior of the rays. We are currently working on the computation of the transverse wave propagation in order to validate the DRT model entirely by comparison to the hybrid code. In order to improve the computation time and the numerical precision, we intend to increase the order of the iterative numerical scheme by using the common fourth-order Runge-Kutta method. Then, the model will be applied to other mocks-up of bimetallic and austenitic welds and to curved composites described with a continuously varying crystallographic orientation.

\section{ACKNOWLEDGMENTS}

The authors want to thank the IRSN (Institut de Radioprotection et de Sureté du Nucléaire) for providing the mock-up of a bimetallic weld on which this work has been performed.

\section{REFERENCES}

1. B. Chassignole, O. Dupond, L. Doudet, V. Duwig, and N. Etchegaray, "Ultrasonic examination of austenitic weld: illustration of the disturbances of the ultrasonic beam," in Review of Progress in Quantitative Nondestructive Evaluation, edited by D. O. Thompson, and C. D. E., 2009, vol. 28, pp. 1886-1893.

2. D. S. Kupperman, and D. E. Reimann, IEEE Transactions on Sonics and Ultrasonics SU-27 no. 1, 7-15 (1980).

3. P. Fellinger, R. Marklein, K. J. Langenberg, and S. Klaholz, Wave Motion 21, 47-66 (1995).

4. A. Apfel, J. Moysan, G. Corneloup, T. Fouquet, and B. Chassignole, Ultrasonics 43, 447-456 (2005).

5. B. Chassignole, V. Duwig, M.-A. Ploix, P. Guy, and R. El Guerjouma, Ultrasonics 49, 653-658 (2009).

6. J. A. Ogilvy, NDT International 18 (2), 67-77 (1985).

7. CIVA software platform for simulating NDT techniques (UT, EC, RT) (2013), URL http: / / www-civa . cea. fr.

8. A. Lhémery, P. Calmon, I. Lecœur-Taïbi, R. Raillon, and L. Paradis, NDT\&E international 33, 499-513 (2000).

9. Plugin OrientationJ (2013), URL http: / / bigwww.epfl.ch/demo/orientation/.

10. P. Thierry, S. Operto, and G. Lambaré, Geophysics 64 (1), 162-181 (1999).

11. V. Červený, Seismic Ray Theory, Cambridge: Cambridge University Press, 2001.

12. G. D. Connolly, Modelling of the propagation of ultrasound through austenitic steel welds, Ph.D. thesis, Imperial College London (2009).

13. S. Mahaut, N. Leymarie, C. Poidevin, T. Fouquet, and O. Dupond, Insight - Non-Destructive Testing and Condition Monitoring 53(12), 664-667 (2011).

14. H. Keers, F. A. Dahlen, and G. Nolet, Geophys. J. Int. 131, 361-380 (1997).

15. K. Žáček, Pure appl. geophys. 159, 1507-1526 (2002).

16. L. Klimeš, Stud. Geophys. Geod. 28, 237-247 (1984). 